\title{
Liver kinase B1 suppresses metastasis and angiogenesis of lung cancer: involvement of the Shh signaling pathway
}

\author{
G. ZHENG ${ }^{1}, \mathrm{~K}$ SONG ${ }^{1}, \mathrm{Y} . \mathrm{ZHAO}^{2, *}$
}

${ }^{1}$ Department of Pathology, College of Basic Medical Sciences, China Medical University, Shenyang 110122, China; ${ }^{2}$ Department of Pathology, College of Basic Medical Sciences and the First Affiliated Hospital, China Medical University, Shenyang 110001, China

${ }^{*}$ Correspondence: zhaoyue@cmu.edu.cn

Received August 20, 2018 / Accepted October 19, 20187

\begin{abstract}
Liver kinase B1 (LKB1) has been shown to be involved in many cancers. However, the underlying mechanism remains unclear. This study aimed to reveal the mechanism through which LKB1 performs its role in lung cancer. LKB1 shRNA and overexpression plasmid were employed to investigate the role of LKB1 in lung cancer in vitro and in vivo. The involvement of Shh signaling pathway was assessed by western blot. Silencing LKB1 promoted migration, invasion and angiogenesis of lung cancer cells, while overexpression of LKB1 led to the opposite. Further study showed that Shh signaling pathway is suppressed by LKB1. Cyclopamine, a Shh signaling pathway inhibitor, reduced the effects of LKB1 silencing, indicating that LKB1 inhibits migration, invasion and angiogenesis through suppressing the Shh signaling pathway. In vivo study also showed that Shh signaling pathway is involved in the modulation of LKB1 in metastasis and angiogenesis of lung cancer. Our study demonstrates that LKB1 inhibits metastasis and angiogenesis of lung cancer through suppressing the Shh signaling pathway.
\end{abstract}

Key words: liver kinase B1, sonic hedgehog, migration, invasion, angiogenesis, lung cancer

Lung cancer, one of the most common malignancies in the worldwide, is generally identified as small cell lung cancer or non-small cell lung cancer (NSCLC). NSCLC includes large cell carcinoma, adenocarcinoma, squamous cell carcinoma and so on, accounting for up to $80-85 \%$ of all lung cancer in clinic $[1,2]$. Lung cancer metastasis, an important event contributing to the deterioration of cancer, is responsible for nearly $90 \%$ of death of lung cancer patients [3].

Liver kinase B1 (LKB1), also known as serine/threonine kinase 11 , is a serine/threonine kinase with a nuclear localization signal and kinase domain. LKB1 is a multifunctional protein implicated in cell proliferation, metastasis, cell polarity and energy metabolism $[4,5]$. AMP-activated protein kinase is the major downstream target, through which LKB1 activates multiple signaling pathways and performs its function. LKB1 is first identified as a causative gene for Peutz-Jeghers syndrome. Peutz-Jeghers syndrome is also associated with cancer development, and therefore LKB1 is supposed to act as a tumor suppressor [6]. LKB1 arrests cell cycle process through p53 and p16 [7], and modulates the epithelial-to-mesenchymal transition (EMT) progress through zinc-finger E-box binding homeobox factor 1 and transforming growth factor- $\beta$ (TGF- $\beta$ ) signaling pathway $[8$,
9]. Indeed, loss of LKB1 promotes the proliferation, migration and angiogenesis of cancer cells $[4,10,11]$. LKB1 is also reported to impact the sensibility of cancer cells to radiotherapy and chemotherapy $[12,13]$.

Although somatic mutation of LKB1 is found in various kinds of cancers $[14,15]$, this phenomenon is rare. Whereas, inactivation of LKB1 due to point mutation or deletion frequently appears in lung cancer, especially in NSCLC, with more than $30 \%$ mutation, ranking the third in lung cancer after TRP53 and Kras $[16,17]$. Interestingly, LKB1 acts as an important barrier in tumorigenesis of lung cancer, controlling its initiation, metastasis and differentiation [17-19]. LKB1 is also reported to suppress the growth and metastasis of lung cancer cells $[19,20]$. However, the underlying mechanism remains unclear and needs to be fully revealed. In the present study, we aimed to investigate how LKB1 influences the metastasis and angiogenesis of lung cancer.

\section{Materials and methods}

Construction of LKB1 overexpression plasmid. Coding sequence of LKB1 was amplified through polymerase chain reaction (PCR) with primers in Table 1. The PCR product 
was linked to pUM-T quick cloning vector (BioTeke, Beijing, China). After sequencing confirmation, the LKB1 coding sequence was cut down and recombined into pcDNA3.1 vector. After sequencing confirmation, this recombinant plasmid was named as LKB1 OE. Blank vector served as negative control of LKB1 OE.

Construction of LKB1 silence plasmid. LKB1 specific shRNA or scramble sequence (Table 1) was synthesized by Sangon Biotech (Shanghai, China) and inserted into pRNA-H1.1/Neo vector. After sequencing confirmation, the recombinant plasmids were named as LKB1 SI and NC, respectively. Scramble sequence served as negative control.

Cell culture. Lung cancer cells A549 and H1299 and human umbilical vein endothelial cells (HUVECs) were obtained from Zhongqiaoxinzhou Biotechnology Co., Ltd (Shanghai, China). These cells were grown in DMEM (Gibco, Grand Island, NY, USA) containing 10\% fetal bovine serum (FBS) (Hyclone, Logan, UT, USA) and cultured in a humid atmosphere at $37^{\circ} \mathrm{C}$ containing $5 \% \mathrm{CO}_{2}$.

Transfection and cyclopamine treatment. Cells were seeded into 6-well plates. When the cell density reached $70 \%$, LKB SI or NC was transfected into H1299 cells, and LKB1 OE or Vector were transfected into A549 cells using Lipofectamine 2000 Reagent (Invitrogen) according to the protocol. Thereafter, the cells were selected using G418 $(500 \mu \mathrm{g} / \mathrm{ml}$; Invitrogen). For cyclopamine treatment, cells were treated with $10 \mu \mathrm{M}$ cyclopamine (Aladdin, Shanghai, China) for $48 \mathrm{~h}$, and then the cells were subjected to wound healing assay, transwell assay and Western blot.

Conditioned medium treatment. After transfection or treatment with $10 \mu \mathrm{M}$ cyclopamine, cells were cultured for additional $48 \mathrm{~h}$. Cell medium was then collected. HUVECs were treated with this conditioned medium for $48 \mathrm{~h}$ and subjected to 3-(4,5-dimethyl-2-thiazolyl)-2,5-diphenyl-2-Htetrazolium bromide (MTT) assay, wound healing assay and tube formation assay.
MTT assay. HUVECs were seeded into 96-well plates $\left(4 \times 10^{3}\right.$ cells/well $)$ and treated with conditioned medium from each group for $48 \mathrm{~h}$. Then MTT at a final concentration of $0.5 \mathrm{mg} / \mathrm{ml}$ was added into each well. After additional culture for $4 \mathrm{~h}$, the supernatant was removed and $150 \mu \mathrm{l}$ of dimethyl sulfoxide (Sigma, St.Louis, MO, USA) was added into each well. The absorbance at $570 \mathrm{~nm}$ was measured with a microplate reader (BIOTEK, Winooski, VT, USA).

Tube formation assay. HUVECs were seeded into a 96-well plate pre-coated with Matrigel (BD Biosciences, San Jose, CA, USA) $\left(1 \times 10^{4}\right.$ cells/well $)$ and treated with conditioned medium for $24 \mathrm{~h}$. Then images of cells were captured with a $100 \times$ magnification.

Enzyme-linked immunosorbent assay (ELISA). After transfection and treatment with cyclopamine, the supernatant of cells was collected. The concentration of vascular endothelial growth factor (VEGF) in the supernatant was measured using a VEGF ELISA kit (Boster, Wuhan, China).

Wound healing assay. Cells were seeded into a 6-well plate. Before wound healing assay, cell medium was changed to fresh serum-free medium and cells were treated with $1 \mu \mathrm{g} / \mathrm{ml}$ mitomycin C (Sigma) for $1 \mathrm{~h}$. Thereafter, scratches were made on monolayer cell surface with $200 \mu$ l pipette tips. After rinsing with serum-free medium, the cells were grown in serum-free medium. Twelve or twenty-four hours later, images of cells were captured with a $100 \times$ magnification. The migration rate was calculated as follows: migration rate $=$ (gap between initial edges - gap between migrated edges) / gap between initial edges $\times 100 \%$.

Transwell assay. The transwell inserts (Corning, Tewksbury, MA, USA) were pro-coated with Matrigel (BD Biosciences). Cells in each group were made into cell suspension in serum-free medium and seeded into transwell inserts ( $2 \times 10^{4}$ cells in $200 \mu \mathrm{l}$ serum-free cell medium). The lower chambers were added with $800 \mu \mathrm{l}$ fresh medium containing $20 \%$ FBS. The cells were allowed to invade for $48 \mathrm{~h}$. There-

Table 1. Primers used for plasmid construction.

\begin{tabular}{lll}
\hline Name & Forward primer & Reverse primer \\
\hline LKB1 OE & 5'-CCGGAATTCATGGAGGTGGTGGACCCGCA-3' & 5'-CCGCTCGAGTCACTGCTGCTTGCAGGCC-3' \\
LKB1 SI & 5'-GATCCCCGCTCTTACGGCAAGGTGAATTCAAGAGA- & 5'-AGCTAAAAAGCTCTTACGGCAAGGTGAATCTCTT- \\
& TTCACCTTGCCGTAAGAGCTTTTT-3' & GAATTCACCTTGCCGTAAGAGCGGG-3' \\
NC forward primer & 5'-GATCCCCTTCTCCGAACGTGTCACGTTTCAAGAGA- & 5'-AGCTAAAAATTCTCCGAACGTGTCACGTTCTCTT- \\
& ACGTGACACGTTCGGAGAATTTTT-3' & GAAACGTGACACGTTCGGAGAAGGG-3' \\
\hline
\end{tabular}

Table 2. Primers for quantitative real-time PCR.

\begin{tabular}{lll}
\hline Gene & Forward primer & Reverse primer \\
\hline Shh & 5'-ACCGAGGGCTGGGACGAAGA-3' & 5'-GATTTGGCCGCCACCGAGTT-3' \\
Ptch & 5'-ACTGGCAGGAGGAGTTGATT-3' & 5'-GTGCTCGTACATTTGCTTGG-3' \\
Smo & 5'-TGCCCTTGGTTCGGACAGAC-3' & 5'-CAAAGAAGCACGCATTGACG-3' \\
Gli1 & 5'-TTCCTACCAGAGTCCCAAGT-3' & 5'-CCCTATGTGAAGCCCTATTT-3' \\
$\beta$-actin & 5'-CTTAGTTGCGTTACACCCTTTCTTG-3' & 5'-CTGTCACCTTCACCGTTCCAGTTT-3' \\
\hline
\end{tabular}


after, the transwell inserts were washed with PBS and fixed with $4 \%$ paraformaldehyde. Cells upon the membranes were removed and cells below the membranes were stained with $0.5 \%$ crystal violet. Cell images were captured with an inverted microscope $(200 \times)$. The average number of cells in 5 random vision fields was used as the number of invading cells.

Western blot. Total proteins were extracted and centrifuged at $10000 \times \mathrm{g}$ at $4^{\circ} \mathrm{C}$. The supernatant was collected and analyzed using a BCA protein assay kit (Beyotime). Then, equal amounts of proteins were separated by sodium dodecyl sulfate-polyacrylamide gel electrophoresis and then transferred onto polyvinylidene fluoride (PVDF) membranes (Merck Millipore, Darmstadt, Germany). After blocking with 5\% defatted milk, the PVDF membranes were incubated with LKB1 antibody (1:500, Boster), E-cadherin antibody (1:500, Cell Signaling Technology, Beverly, MA, USA), N-cadherin antibody (1:500, Cell Signaling Technology), Vimentin antibody (1:1000, Cell Signaling Technology), Shh antibody (1:500, Bioss, Beijing, China), patched (Ptch) antibody (1:500, Proteintech, Wuhan, China), smoothened (Smo) antibody (1:1000, Abgent, Suzhou, China), glioma-associated oncogene homologue 1 (Gli1) antibody (1:1 000, Sangon Biotech), metal matrix proteinase (MMP)-2 antibody (1:1000, Sangon Biotech), MMP-9 antibody (1:1000, Sangon Biotech), VEGF antibody (1:1000; Abcam, Cambridge, UK), cyclinD1 antibody (1:1000, Bioss), Bcl-2 antibody (1:1000, Bioss) or $\beta$-actin antibody (1:500, Bioss) at $4^{\circ} \mathrm{C}$ overnight. Then the PVDF membranes were rinsed with tris buffered saline with Tween (TBST) and incubated with horseradish peroxidase (HRP)-labeled secondary antibodies (1:5000, Beyotime) at $37^{\circ} \mathrm{C}$ for $45 \mathrm{~min}$. Thereafter, the PVDF membranes were rinsed with TBST and visualized with an ECL kit (Beyotime). The gray levels of targeted bands were analyzed with Gel-Pro-Analyzer.

Quantitative real-time PCR. Total RNA in cells was extracted using TRIpure (BioTeke) according to the manufacturer's protocol. Total RNA was reversely transcribed to cDNA using Super M-MLV (BioTeke) and Oligo $(\mathrm{dT})_{15}$ according to the protocol. Then the mRNA levels of Shh, Ptch, Smo and Gli1 were measured through quantitative real-time PCR using cDNA as templates and primers in Table 2. Relative mRNA level was calculated using $2^{-\Delta \Delta \mathrm{Ct}}$ method.

Immunofluorescence. Cells were fixed with $4 \%$ paraformaldehyde and permeabilized with $0.1 \%$ TritonX-100. After rinsing with PBS, cells were blocked with goat serum (Solarbio, Beijing, China) at room temperature for 15 min. Then cells were incubated with Gli1 antibody (1:100, Abclonal, Cambridge, MA, USA) at $4{ }^{\circ} \mathrm{C}$ overnight. After rinsing with $\mathrm{PBS}$, cells were incubated with Cy3-labeled secondary antibody (1:200, Beyotime) at room temperature for $60 \mathrm{~min}$. After rinsing with PBS, the cells were incubated with DAPI and observed with a fluorescence microscope (OLYMPUS, Tokyo, Japan). Images of cells were obtained under a 400x magnification.
Animal experimental protocol. This study was carried out according to the Guide for Care and Use of Laboratory Animals, and approved by the Ethics Committee of China Medical University. Healthy nude mice (4-6 weeks old, 18-20 g) were obtained from Huafukang Bioscience Co. Inc (Beijing, China). The mice were fed in a standard environment $\left(25 \pm 1^{\circ} \mathrm{C}, 55-65 \%\right.$ humidity, $12 \mathrm{~h}$ light $/ 12 \mathrm{~h}$ dark cycles) for 1 week to adapt, and then randomly divided into 3 groups: NC group, LKB1 SI group, and LKB1 SI+cyclopamine group. $\mathrm{N}=6$ for each group. Mice in NC group received an injection of NC cells $\left(2 \times 10^{6}\right.$ cells in $\left.200 \mu \mathrm{PBS}\right)$ through caudal vein. Mice in LKB1 SI group received an injection of LKB SI cells $\left(2 \times 10^{6}\right.$ cells; caudal vein injection). Mice in LKB1 SI+cyclopamine group received an injection of LKB SI cells $\left(2 \times 10^{6}\right.$ cells; caudal vein injection) and injections of cyclopamine $(25 \mathrm{mg} / \mathrm{kg} /$ day; intraperitoneal injection). Sixty days later, the mice were sacrificed and their lung tissues were obtained for subsequent hematoxylin and eosin (HE) staining and immunohistochemistry.

HE staining. Lung tissues were fixed with $4 \%$ paraformaldehyde. After dehydration in gradient ethanol, lung tissues were embedded into paraffin and cut into $5 \mu \mathrm{m}$ slices. After dewaxing and rehydrating, slices were stained with hematoxylin and eosin. Images were captured with a $40 \times$ magnification.

Immunohistochemistry. Slices were rehydrated in gradient ethanol and retrieved in antigen retrieval buffer. Endogenous peroxidases were inactivated with 3\% hydrogen peroxide. Slices were blocked with goat serum and then incubated with CD31 antibody $(1: 100 ; \mathrm{Abcam})$ at $4{ }^{\circ} \mathrm{C}$ overnight. After rinsing in PBS, slices were incubated with biotin-conjugated secondary antibodies (1:200; Beyotime) at $37^{\circ} \mathrm{C}$ for $30 \mathrm{~min}$. Then slices were rinsing with PBS and incubated with HRP-conjugated avidin (1:200; Beyotime) at $37^{\circ} \mathrm{C}$ for $30 \mathrm{~min}$. DAB was used for visualization of targeted protein and hematoxylin was used for cell nucleus counterstaining. Images were obtained with a $400 \times$ magnificationion.

Statistical analysis. Experiments were repeated for three times. All the results were presented as mean \pm SD. Differences between each group were analyzed using One-way Analysis of Variance or Student's t test. A p $<0.05$ was considered as significant.

\section{Results}

LKB1 suppresses the migration and invasion of lung cancer cells in vitro. In our study, a LKB1 specific shRNA was used to downregulate the level of LKB1 and a LKB1 overexpression plasmid was constructed to upregulate the level of LKB1. Then the efficiency of LKB1 shRNA and LKB1 overexpression plasmid was verified by western blot. Level of LKB1 was downregulated by LKB1 shRNA and upregulated by LKB1 overexpression plasmid (Supplementary Figures $1 \mathrm{~A}$ and $\mathrm{B})$. These results demonstrate that LKB1 shRNA downregulates and LKB1 overexpression plasmid upregulates the level of LKB1 effectively. 
Then migration capability was assessed by wound healing assay. After silence of LKB1, the cell migration capability was increased (Figure 1A); while, after LKB1 overexpression, the migration capability was decreased (Figure 1B). Meanwhile, the invasion capability was evaluated by transwell assay. Number of invading cells was increased after LKB1 silence (Figure 1C) and decreased after LKB1 overexpression (Figure 1D). These results suggest that LKB1 suppresses the migration and invasion of lung cancer cells.

EMT is an important event impacting the metastasis of cancer cells. In the present study, levels of EMT-related proteins were also detected by western blot. The level of E-cadherin was decreased by LKB1 silence and increased by LKB1 overexpression. Whereas, the level of $\mathrm{N}$-cadherin was increased by LKB1 silence and declined by LKB1 overexpression (Figures 1E-H). Also, the level of Vimentin was increased by LKB1 silence and decreased by LKB1 overexpression (Figures $1 \mathrm{E}-\mathrm{H}$ ). These results indicate that LKB1 also suppresses the EMT process of lung cancer cells.

LKB1 inhibits angiogenesis. After transfection, conditioned medium of lung cancer cells was harvested. HUVECs were treated with this conditioned medium and then the angiogenesis of HUVECs were evaluated by MTT assay, wound healing assay and tube formation assay. The results of our study showed that LKB1 silence increased cell viability and migration of HUVECs, and increased their angiogenesis. Whereas, overexpression of LKB1 inhibited cell viability and migration of HUVECs, and decreased their angiogenesis capability (Figures 2A-E). Moreover, the VEGF level in cell medium and in lung cancer cells was measured by ELISA assay and western blot, respectively. After silence of LKB1, the VEGF concentration in cell medium and VEGF protein level in lung cancer cells were both increased. While, after overexpression of LKB1, the VEGF level in cell medium and lung cancer cells was decreased (Figures 2F and G). These results demonstrate that $\mathrm{LKB} 1$ inhibits angiogenesis.

LKB1 suppresses the migration, invasion and angiogenesis of lung cancer cells in vitro through suppressing the Shh signaling pathway. In our study, we also investigated whether Shh signaling pathway was involved in the effect of LKB1. Our results showed that, after silence of LKB1, the mRNA levels of Shh, Ptch, Smo and Gli1, key molecules in Shh signaling pathway, were increased; whereas, after overexpression of LKB1, the mRNA levels of Shh, Ptch, Smo and

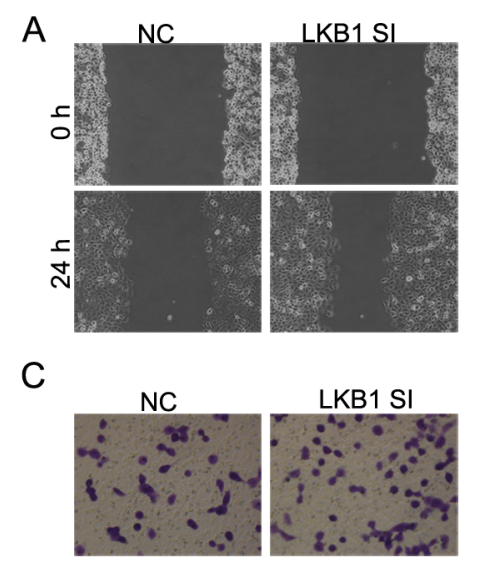

$E$

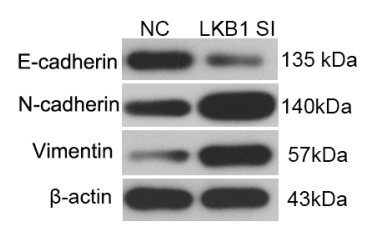

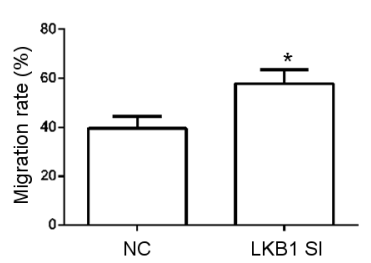

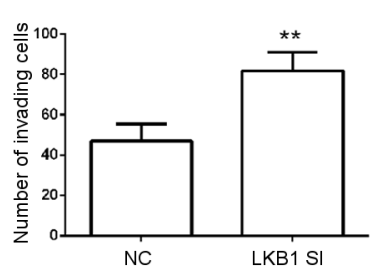

$\mathrm{F}$

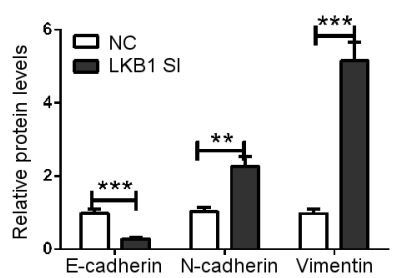

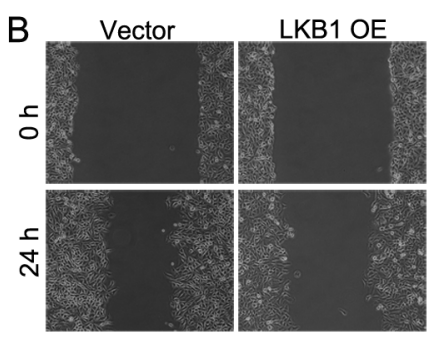

D
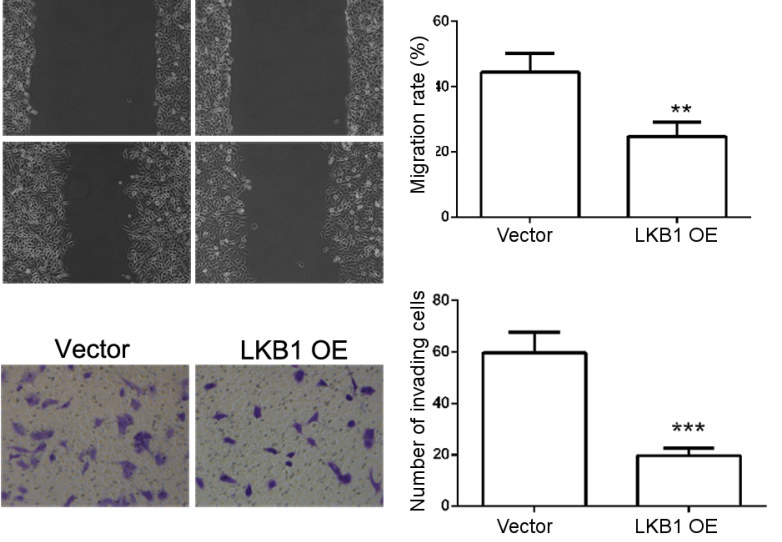

G

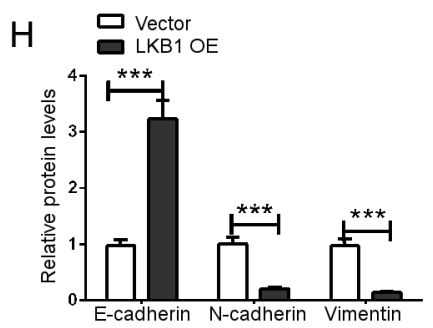

Figure 1. LKB1 inhibits the migration, invasion and EMT of lung cancer cells. A) After silence of LKB1, the migration capability of H1299 cells was assessed by wound healing assay. B) After overexpression of LKB1, wound healing assay was carried out to assess the migration capability of A549 cells. C) Transwell assay was performed to detect the invasive capability of H1299 cells after LKB1 silence. D) After overexpression of LKB1 in A549 cells, the invasive capability was assessed by transwell assay. E and F) After silence of LKB1, protein levels of E-cadherin, N-cadherin and vimentin in H1299 cells were detected by Western blot with $\beta$-actin as the internal control. G and $\mathrm{H}$ ) Western blot was carried out to detect the protein levels of E-cadherin, $\mathrm{N}$-cadherin and vimentin in A549 cells after LKB1 overexpression. Each experiment was repeated for three times. The results were presented as mean \pm SD. ${ }^{\star} \mathrm{p}<0.05,{ }^{\star *} \mathrm{p}<0.01,{ }^{\star * \star} \mathrm{p}<0.001$. EMT, epithelial-to-mesenchymal transition; LKB1 SI, LKB1 silence; NC, negative control of LKB1 silence; LKB1 OE, LKB1 overexpression; Vector, negative control of LKB1 overexpression. 

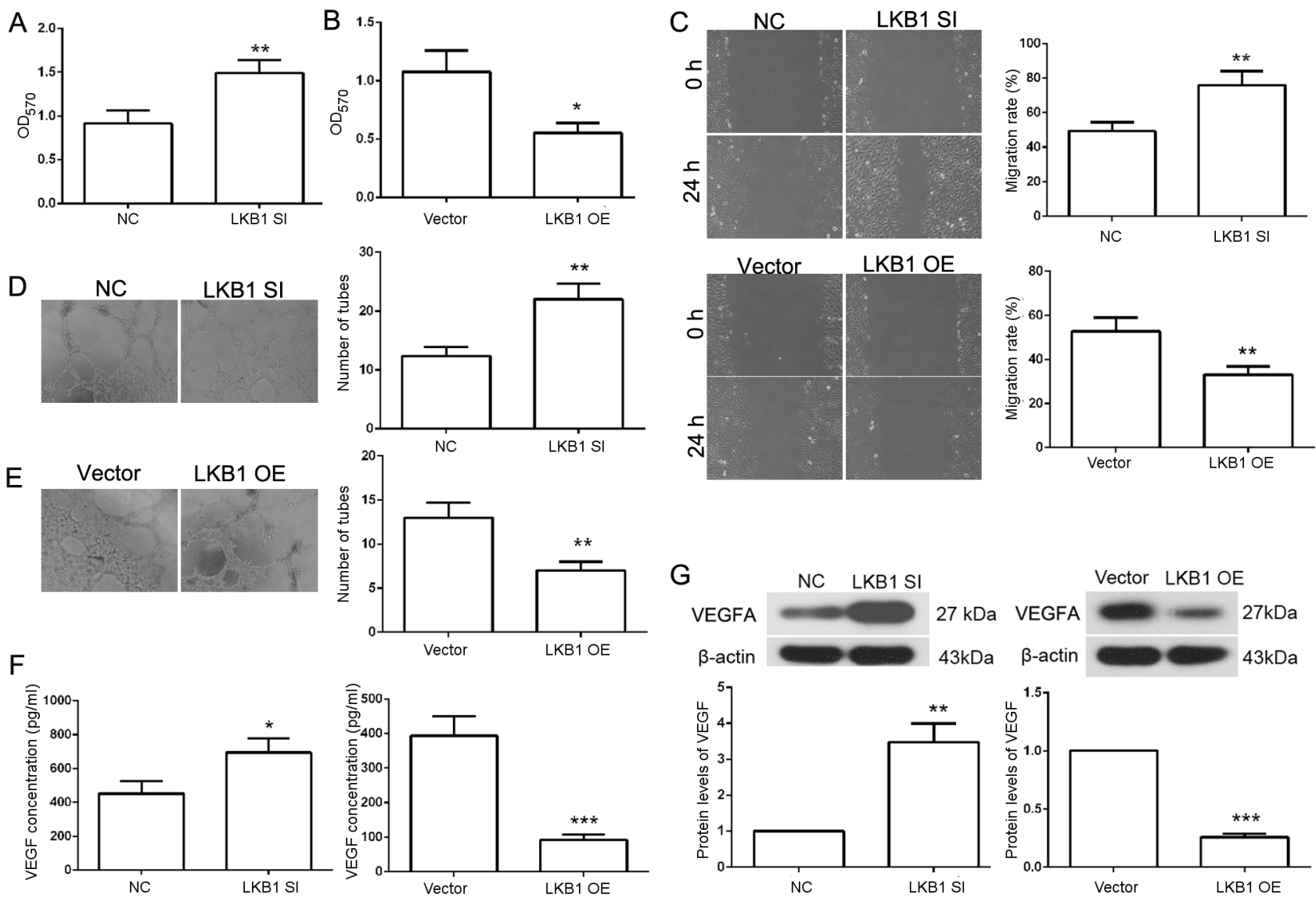

Figure 2. LKB1 inhibits angiogenesis. A and B) After treatment with conditioned medium, cell viability of HUVECs was assessed by MTT assay. C) Wound healing assay was performed to assess the migration capability of HUVECs after treatment with conditioned medium. D and E) Tube formation assay of HUVECs was performed after treatment with conditioned medium. F) The concentration of VEGF in cell medium of H1299 cells and A549 cells was measured by ELISA. G) The level of VEGF in H1299 cells and A549 cells was detected by Western blot. All experiments were repeated for three times. The results were presented as mean $\pm \mathrm{SD} .{ }^{\star} \mathrm{p}<0.05,{ }^{\star *} \mathrm{p}<0.01,{ }^{\star *} \mathrm{p}<0.001$. LKB1 SI, LKB1 silence; NC, negative control of LKB1 silence; LKB1 $\mathrm{OE}, \mathrm{LKB} 1$ overexpression; Vector, negative control of LKB1 overexpression.

Gli1 were decreased (Figures $3 \mathrm{~A}$ and B). The results of western blot assay showed similar results. The protein levels of Shh, Ptch, Smo and Gli1 were raised by LKB1 silence and declined by LKB1 overexpression (Figures 3C-F). Consistently, the levels of cyclinD1 and Bcl-2, target genes of Gli1, were also raised by LKB1 silence and decreased by LKB1 overexpression (Figures 3C-F). Nuclear translocation of Gli1 was evaluated by immunofluorescence with Glil antibody. Our results showed that, after LKB1 overexpression, nuclear translocation of Gli1 was reduced, whereas, after LKB1 silence, the nuclear translocation of Gli1 was enhanced (Figures 3G). These results demonstrate that the Shh signaling pathway was inhibited by LKB1.

As Shh signaling pathway was impacted by LKB1, cyclopamine, an inhibitor of Shh signaling pathway, was employed in our study. The effectiveness of cyclopamine was shown in Supplementary Figures 1C-F. After treatment with cyclopamine, the enhanced migration of lung cancer cells induced by LKB1 SI was reduced (Figure 4A). Consistently, the enhanced invasion of lung cancer cells induced by LKB1 silence was reduced after cotreatment with cyclopamine (Figure 4B). These results suggest that LKB1 suppresses the migration and invasion of lung cancer cells through inhibiting the Shh signaling pathway. To further provide evidence for our hypothesis, the levels of E-cadherin, N-cadherin, Vimentin, MMP-2 and MMP-9 were also detected by western blot assay after co-treatment with cyclopamine. The level of E-cadherin was decreased, and the levels of N-cadherin, Vimentin, MMP-2 and MMP-9 were increased by LKB1 silence. Whereas, these effects of LKB1 silence were reduced by co-treatment with cyclopamine (Figures 4C and D). These results provide further evidence for our hypothesis that LKB1 suppresses the migration and invasion of lung cancer cells through suppressing the Shh signaling pathway.

VEGF concentration in cell medium and VEGF protein level in lung cancer cells were also measured by ELISA assay and western blot assay, respectively. The increased concentration of VEGF in cell medium and protein level of VEGF in 

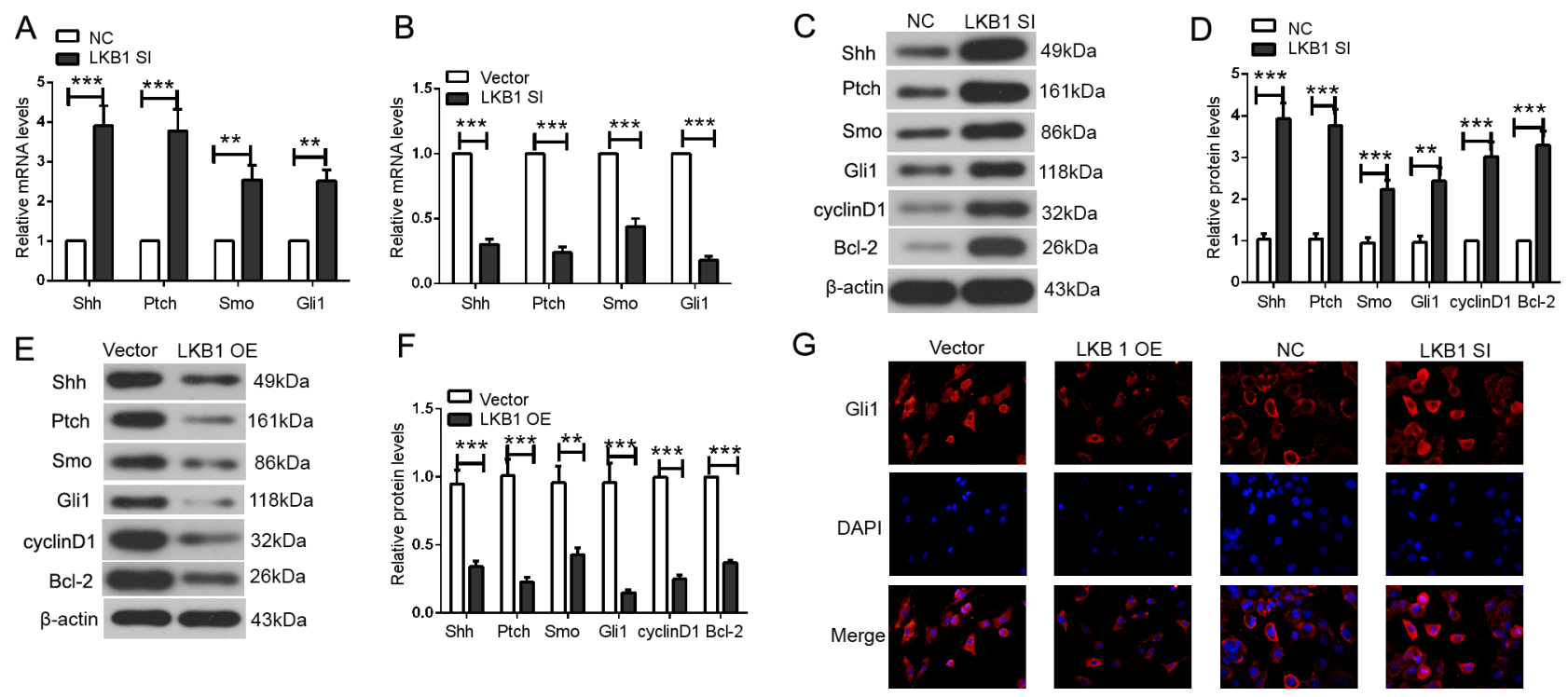

Figure 3. LKB1 inhibits the Shh signaling pathway. A) The mRNA levels of Shh, Ptch, Smo and Gli1 in H1299 cells were measured by quantitative real-time PCR. Relative mRNA level was calculated using $2^{-\Delta \Delta C t}$ method. B) Quantitative real-time PCR was performed to measure the mRNA levels of Shh, Ptch, Smo and Gli1. C and D) After silence of LKB1, the protein levels of Shh, Ptch, Smo, Gli1, cyclinD1 and Bcl-2 in H1299 cells were detected by Western blot. $\beta$-actin served as the internal control. E and F) Western blot was carried out to detect the protein levels of Shh, Ptch, Smo, Gli1, cyclin D1 and Bcl-2 in A549 cells after LKB1 overexpression. G) Immunofluorescence was performed to detect the expression and distribution of Gli1. All experiments were repeated for three times. The results were presented as mean \pm SD. ${ }^{\star *} p<0.01,{ }^{\star *}$ p $<0.001$. LKB1 SI, LKB1 silence; NC, negative control of LKB1 silence; LKB1 OE, LKB1 overexpression; Vector, negative control of LKB1 overexpression.

lung cancer cell induced by LKB1 SI was reduced by co-treatment with cyclopamine (Figures $4 \mathrm{E}$ and F). These results suggest that LKB1 inhibits the angiogenesis through the Shh signaling pathway.

LKB1 suppresses the metastasis and angiogenesis of lung cancer in vivo through suppressing the Shh signaling pathway. We also verified our hypothesis in an in vivo study. Lung cancer cells were inoculated into nude mice through caudal vein. Two months later, the tumor nodes in lung tissues were observed through $\mathrm{HE}$ staining. The increased number of tumor nodes induced by LKB1 silence was reduced by injection of cyclopamine (Figures $5 \mathrm{~A}$ and B). Moreover, angiogenesis was assessed by immunohistochemistry with a CD31 antibody. The results showed that the elevated vessels induced by LKB1 silence were reversed by co-treatment with cyclopamine (Figure 5C). These results suggest that LKB1 suppresses the metastasis and angiogenesis of lung cancer in vivo through suppressing the Shh signaling pathway.

\section{Discussion}

In our study, we found that silencing LKB1 promoted, while overexpression of LKB1 inhibited migration, invasion and angiogenesis of lung cancer cells. Further study showed that Shh signaling pathway was suppressed by LKB1. Shh signaling pathway inhibitor, cyclopamine, reduced the effects of LKB1 silencing, indicating that LKB1 may inhibit migration, invasion and angiogenesis of lung cancer cells through suppressing the Shh signaling pathway. Our in vivo study also showed that Shh signaling pathway was involved in the LKB1 effects on metastasis and angiogenesis of lung cancer. Our study provides additional information about the possibility for LKB1 to be a potential therapeutic target for lung cancer treatment.

Metastasis frequently contributes to high mortality of lung cancer. LKB1, which is the third mutated gene in lung cancer, is associated with poor prognosis of lung cancer patients [21]. $\mathrm{LKB} 1$, which is commonly regarded as a tumor suppressor, is reported to be negatively associated with the cancer metastasis [4]. Loss of LKB1 confers invasion and metastasis behavior in mouse models of cancer [22]. In our study, we found that silencing LKB1 promoted migration and invasion of lung cancer cells, whereas overexpression of LKB1 inhibited these processes. These results indicate that LKB1 may suppress the lung cancer metastasis. Our in vivo study also showed that metastasis of lung cancer was increased by LKB1 silencing. These results provide further evidence for our hypothesis that LKB1 suppresses the lung cancer metastasis in vitro and in vivo. Retrospective study of Liu et al showed that the level of LKB was negatively correlated to lymph node metastasis [23] . Ji et al also indicated that LKB1 was a critical barrier for the initiation and metastasis of lung cancer [17]. Given that LKB1 may perform a tumor-suppressor role in lung cancer metastasis, LKB1 has the potential to become a target in the lung cancer treatment. 
A
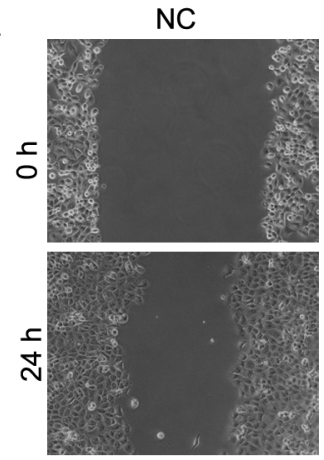

B

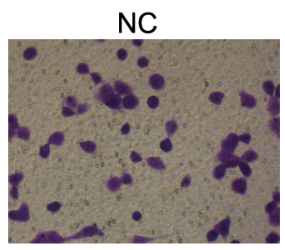

LKB SI
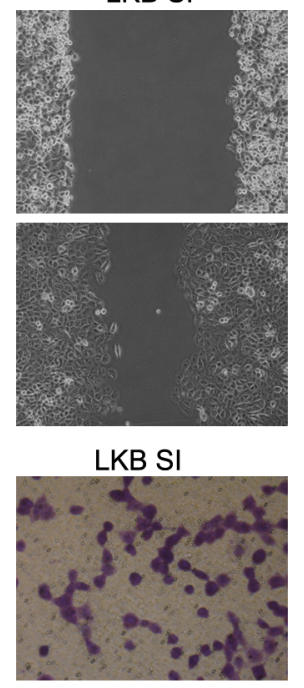

C

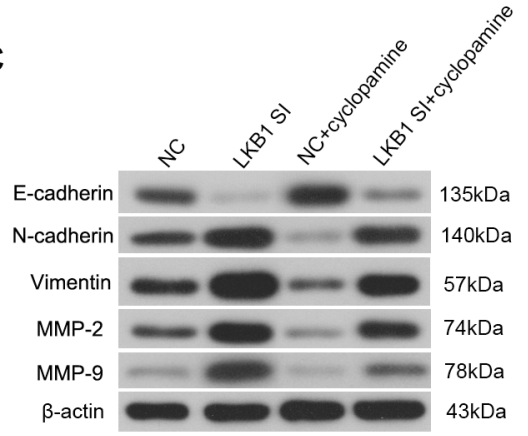

E

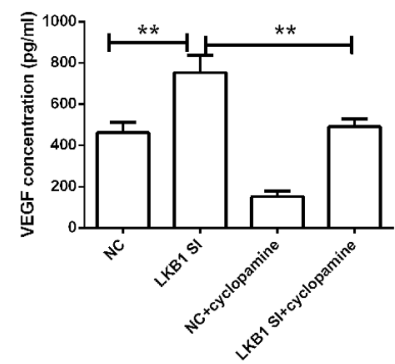

$\mathrm{NC}+$ cyclopamine
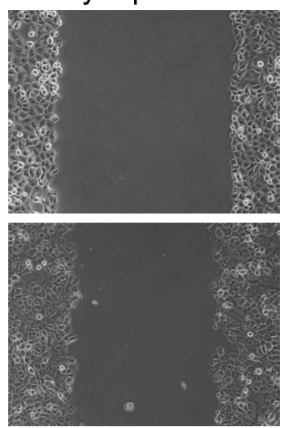

$\mathrm{NC}+$ cyclopamine

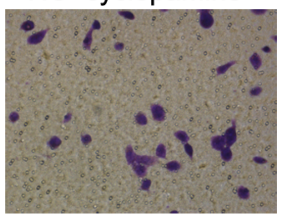

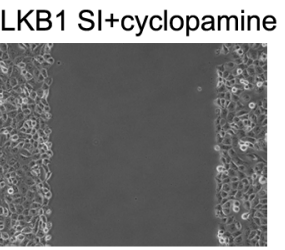

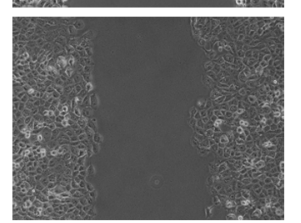

LKB1 SI+cyclopamine

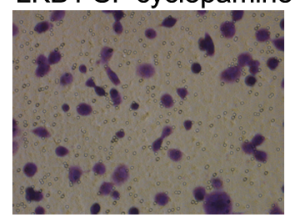

o

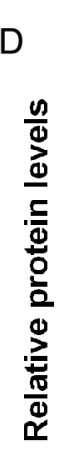

$\square \mathrm{NC}$

$\square$ LKB1 SI

$\square \mathrm{NC}+$ cyclopamine

LKB1 SI+cyclopamine
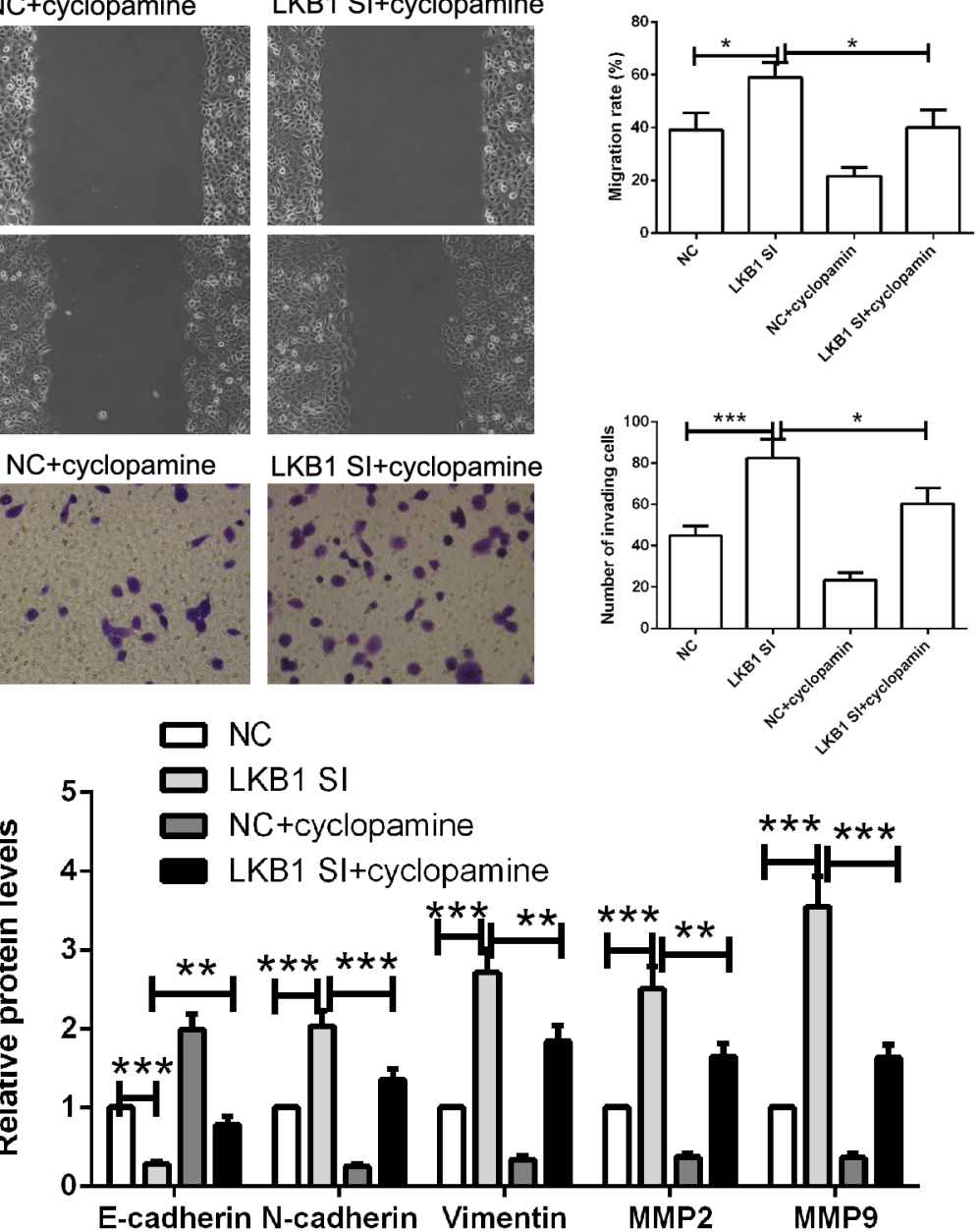
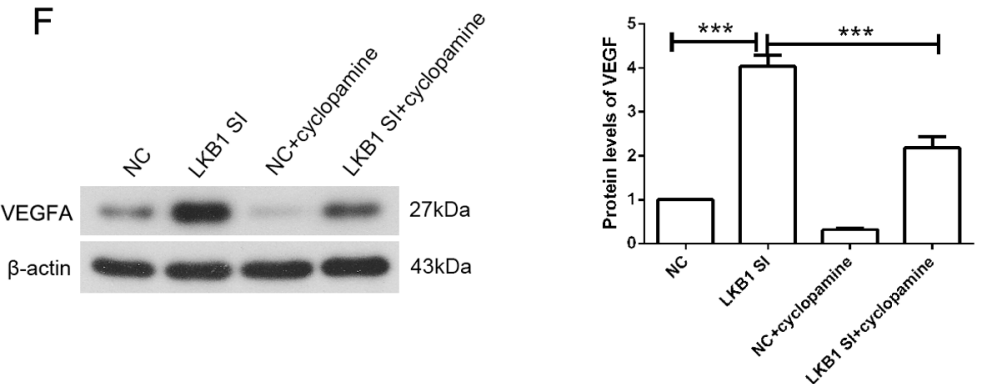

Figure 4. Blocking Shh signaling pathway with cyclopamine reduced the effects of LKB1 SI. After silence of LKB1 and/or treatment with cyclopamine, the migration and invasion capabilities of $\mathrm{H} 1299$ cells were evaluated by wound healing assay (A) and transwell assay (B).The protein levels of Ecadherin, N-cadherin, Vimentin, MMP-2 and MMP-9 in H1299 cells were detected by Western blot with $\beta$-actin as the internal control (C and D). The concentration of VEGF in cell medium of $\mathrm{H} 1299$ cells was measured by ELISA (E) and the level of VEGF in H1299 cells was assessed by Western blot (F). Each experiment was repeated for three times. The results were presented as mean $\pm \mathrm{SD}$. ${ }^{\star} \mathrm{p}<0.05,{ }^{\star *} \mathrm{p}<0.01,{ }^{* *} \mathrm{p}<0.001$. LKB1 SI, LKB1 silence; NC, negative control of LKB1 silence; LKB1 OE, LKB1 overexpression; Vector, negative control of LKB1 overexpression.

MMPs, which degrade basement membrane, play an important role in the migration and invasion of cancer cells. In our study, the MMP-2 and MMP-9 levels were also elevated by LKB1 silencing, which provides further evidence for the LKB1 involvement in lung cancer cell migration and invasion. Consistently, another report also showed that LKB1 down-regulated the MMP expression [24]. MMPs are also closely related to EMT process [25]. The modulation of MMPs by LKB1 indicates a potential role of LKB1 in EMT process of lung cancer. 
A

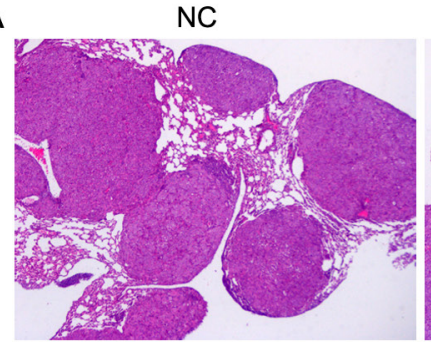

C

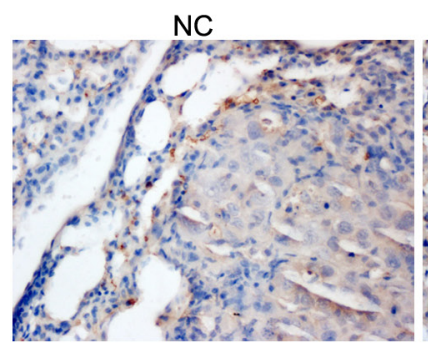

LKB1 SI

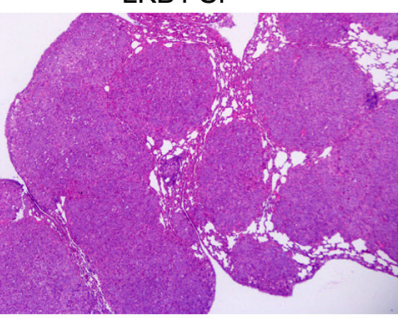

LKB1 SI

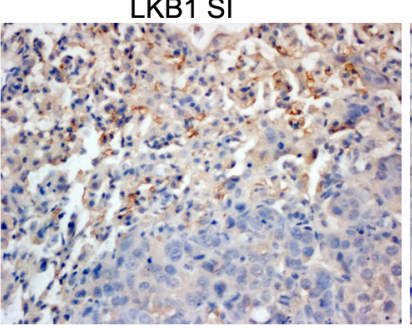

LKB1 SI+cyclopamine

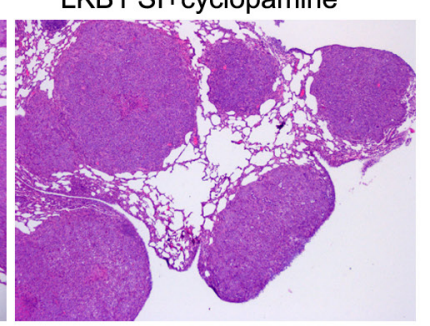

LKB1 SI+cyclopamine

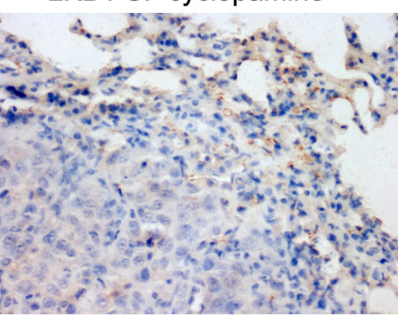

B

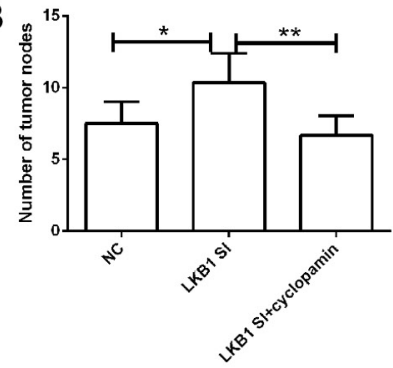

Figure 5. Cyclopamine reduced the effects of LKB1 SI in vivo. A) After silence of LKB1, H1299 cells were injected into nude mice through caudal vein. Mice received cyclopamine $(25 \mathrm{mg} / \mathrm{kg})$ treatment for 60 days. Mice were sacrificed and their lung tissues were stained with $\mathrm{HE}$ staining ( $\mathrm{n}=6)$. B) Number of tumor nodes was counted. C) Expression and distribution of CD31 was evaluated by immunohistochemistry. The results were presented as mean \pm SD. ${ }^{\star} \mathrm{p}<0.05,{ }^{* *} \mathrm{p}<0.01$. LKB1 SI, LKB1 silence; NC, negative control of LKB1 silence.

EMT is an important event in development and it is a complicated program contributing to tumor metastasis. Through EMT program, differentiated cancer cells (epithelial) are reversed to dedifferentiated cancer cells (mesenchymal), vesting cancer cells motility and many attributes of stem cells $[26,27]$. In our study, LKB1 upregulated levels of E-cadherin (an epithelial marker) and downregulated levels of $\mathrm{N}$-cadherin and Vimentin (mesenchymal markers), indicating that LKB1 may repress EMT process in lung cancer cells. Consistently, the report of Roy et al also showed that LKB1 knock-down increased motility and invasiveness of lung cancer cells, accompanied by increased zinc-finger $\mathrm{E}$ box-binding homeobox factor 1, an EMT inducer [8].

Angiogenesis is a crucial event in tumor progression. Angiogenesis provides nutrition for the growing cancer cells and also provides ways for their distant metastases. LKB1 deficiency was also reported to lead to an increase in the vascular endothelial growth factor level, an important growth factor promoting angiogenesis [28]. Consistently, in our study angiogenesis was inhibited by LKB1, with declined VEGF level.

Shh signaling pathway is initiated by Shh binding to Ptch, de-repressing Smo, and finally activating Gli and target genes. It is closely related to cancer initiation and development [29]. Silencing of Shh was found to suppress migration and invasion of cervical cancer, bladder cancer and gastric cancer [30-32], and also to regulate EMT process [33]. Zhuang et al showed that in breast cancer, the LKB1 level was negatively correlated with the expression of transcription factors in Shh signaling pathway [34]. Thus we speculate that the Shh signaling pathway may be involved in the effect of LKB1 on lung cancer. Other hedgehog factors, such as Sufu and Hip, were also impacted by LKB1 [34]. In our study, LKB1 decreased key molecules in Shh signaling pathway. Moreover, treatment with cyclopamine, a Shh signaling pathway inhibitor, reduced the effects of LKB1 silencing, demonstrating that the Shh signaling pathway is involved in the LKB1 role in lung cancer. Other signaling pathways may be also implicated in the effects of LKB1 on lung cancer, and further exploration is needed.

In the present study, we investigated the role of LKB1 in lung cancer. Our study shows that LKB1 inhibited metastasis and angiogenesis of lung cancer. Moreover, Shh signaling pathway was implicated in the effect of LKB1 on lung cancer. As mutation of LKB1 in lung cancer appears up to $30 \%$, LKB1 may become a therapeutic target for the lung cancer treatment.

Supplementary information is available in the online version of the paper.

Acknowledgements: This work was supported by the Natural Science Foundation of Department of Science and Technology, Liaoning Province under Grant (No. 2015204099).

\section{References}

[1] INAMURA K, ISHIKAWA Y. MicroRNA In Lung Cancer: Novel Biomarkers and Potential Tools for Treatment. J Clin Med 2016; 5: E36. https://doi.org/10.3390/jcm5030036 
[2] GONZALEZ-SANCHEZ E, MARTIN-CABALLERO J, FLORES JM, HERNANDEZ-LOSA J, MONTERO MA et al. Lkb1 loss promotes tumor progression of BRAF(V600E)induced lung adenomas. PLoS One 2013; 8: e66933. https:// doi.org/10.1371/journal.pone.0066933

[3] CHAFFER CL, WEINBERG RA. A perspective on cancer cell metastasis. Science 2011; 331: 1559-1564. https://doi. org/10.1126/science. 1203543

[4] LI J, LIU J, LI P, MAO X, LI W et al. Loss of LKB1 disrupts breast epithelial cell polarity and promotes breast cancer metastasis and invasion. J Exp Clin Cancer Res 2014; 33: 70. https://doi.org/10.1186/s13046-014-0070-0

[5] SHAN T, XIONG Y, KUANG S. Deletion of Lkb1 in adult mice results in body weight reduction and lethality. Sci Rep 2016; 6: 36561. https://doi.org/10.1038/srep36561

[6] VAAHTOMERI K, MAKELA TP. Molecular mechanisms of tumor suppression by LKB1. FEBS Lett 2011; 585: 944-951. https://doi.org/10.1016/j.febslet.2010.12.034

[7] LIANG X, WANG P, GAO Q, XIANG T, TAO X. Endogenous LKB1 knockdown accelerates $\mathrm{G}(1) / \mathrm{S}$ transition through p53 and p16 pathways. Cancer Biol Ther 2010; 9: 156-160.

[8] ROY BC, KOHNO T, IWAKAWA R, MORIGUCHI T, KIYONO $\mathrm{T}$ et al. Involvement of LKB1 in epithelial-mesenchymal transition (EMT) of human lung cancer cells. Lung Cancer 2010; 70: 136-145. https://doi.org/10.1016/j.lungcan.2010.02.004

[9] HONG B, ZHANG J, YANG W. Activation of the LKB1SIK1 signaling pathway inhibits the TGFbetamediated epithelialmesenchymal transition and apoptosis resistance of ovarian carcinoma cells. Mol Med Rep 2018; 17: 2837-2844. https://doi.org/10.3892/mmr.2017.8229

[10] WANG YQ, DAI WM, CHU XY, YANG B, ZHAO M et al. Downregulation of LKB1 suppresses Stat3 activity to promote the proliferation of esophageal carcinoma cells. Mol Med Rep 2014; 9: 2400-2404. https://doi.org/10.3892/ mmr.2014.2071

[11] ZHANG W, DING Y, ZHANG C, LU Q, LIU Z et al. Deletion of endothelial cell-specific liver kinase B1 increases angiogenesis and tumor growth via vascular endothelial growth factor. Oncogene 2017; 36: 4277-4287. https://doi. org/10.1038/onc.2017.61

[12] YAO YH, CUI Y, QIU XN, ZHANG LZ, ZHANG W et al. Attenuated LKB1-SIK1 signaling promotes epithelial-mesenchymal transition and radioresistance of non-small cell lung cancer cells. Chin J Cancer 2016; 35: 50. https://doi. org/10.1186/s40880-016-0113-3

[13] OU W, YE S, YANG W, WANG Y, MA Q et al. Enhanced antitumor effect of cisplatin in human NSCLC cells by tumor suppressor LKB1. Cancer Gene Ther 2012; 19: 489-498. https://doi.org/10.1038/cgt.2012.18

[14] WINGO SN, GALLARDO TD, AKBAY EA, LIANG MC, CONTRERAS CM et al. Somatic LKB1 mutations promote cervical cancer progression. PLoS One 2009; 4: e5137. https://doi.org/10.1371/journal.pone.0005137

[15] YALNIZ Z, TIGLI H, TIGLI H, SANLI O, DALAY $\mathrm{N}$ et al. Novel mutations and role of the LKB1 gene as a tumor suppressor in renal cell carcinoma. Tumour Biol 2014; 35: 12361-12368. https://doi.org/10.1007/s13277-014-2550-4
[16] MATSUMOTO S, IWAKAWA R, TAKAHASHI K, KOHNO T, NAKANISHI Y et al. Prevalence and specificity of LKB1 genetic alterations in lung cancers. Oncogene 2007; 26: 5911-5918. https://doi.org/10.1038/sj.onc.1210418

[17] JI H, RAMSEY MR, HAYES DN, FAN C, MCNAMARA K et al. LKB1 modulates lung cancer differentiation and metastasis. Nature 2007; 448: 807-810. https://doi.org/10.1038/ nature 06030

[18] TAJIRI M, WAKABAYASHI N, TSUJINO M, FUJII M, OKABE $\mathrm{K}$ et al. Alterations of the LKB1 gene in lung adenocarcinomas induced by $\mathrm{N}$-nitrosobis(2-hydroxypropyl) amine in rats. Pathobiology 2010; 77: 225-229. https://doi. org/10.1159/000305355

[19] JIMENEZ AI, FERNANDEZ P, DOMINGUEZ O, DOPAZO A, SANCHEZ-CESPEDES M. Growth and molecular profile of lung cancer cells expressing ectopic LKB1: downregulation of the phosphatidylinositol 3'-phosphate kinase/ PTEN pathway. Cancer Res 2003; 63: 1382-1388.

[20] CARRETERO J, SHIMAMURA T, RIKOVA K, JACKSON AL, WILKERSON MD et al. Integrative genomic and proteomic analyses identify targets for Lkb1-deficient metastatic lung tumors. Cancer Cell 2010; 17: 547-559. https://doi. org/10.1016/j.ccr.2010.04.026

[21] JIANG L, LIANG X, LIU M, WANG W, MA J et al. Reduced expression of liver kinase B1 and Beclin1 is associated with the poor survival of patients with non-small cell lung cancer. Oncol Rep 2014; 32: 1931-1938. https://doi.org/10.3892/ or.2014.3432

[22] CONTRERAS CM, AKBAY EA, GALLARDO TD, HAYNIE JM, SHARMA S et al. Lkb1 inactivation is sufficient to drive endometrial cancers that are aggressive yet highly responsive to mTOR inhibitor monotherapy. Dis Model Mech 2010; 3: 181-193. https://doi.org/10.1242/dmm.004440

[23] LIU S, MIAO Y, FAN C, LIU Y, YU J et al. Clinicopathologic correlations of liver kinase B1, E-cadherin, and N-cadherin expression in non-small cell lung cancer. Appl Immunohistochem Mol Morphol 2013; 21: 334-340. https://doi. org/10.1097/PAI.0b013e31826b128b

[24] ZHUANG ZG, DI GH, SHEN ZZ, DING J, SHAO ZM. Enhanced expression of LKB1 in breast cancer cells attenuates angiogenesis, invasion, and metastatic potential. Mol Cancer Res 2006; 4: 843-849. https://doi.org/10.1158/1541-7786. MCR-06-0118

[25] RADISKY ES, RADISKY DC. Matrix metalloproteinaseinduced epithelial-mesenchymal transition in breast cancer. J Mammary Gland Biol Neoplasia 2010; 15: 201-212. https:// doi.org/10.1007/s10911-010-9177-x

[26] MANI SA, GUO W, LIAO MJ, EATON EN, AYYANAN $A$ et al. The epithelial-mesenchymal transition generates cells with properties of stem cells. Cell 2008; 133: 704-715. https://doi.org/10.1016/j.cell.2008.03.027

[27] POLYAK K, WEINBERG RA. Transitions between epithelial and mesenchymal states: acquisition of malignant and stem cell traits. Nat Rev Cancer 2009; 9: 265-273. https://doi. org/10.1038/nrc2620

[28] YLIKORKALA A, ROSSI DJ, KORSISAARI N, LUUKKO $\mathrm{K}$, ALITALO $\mathrm{K}$ et al. Vascular abnormalities and deregulation of VEGF in Lkb1-deficient mice. Science 2001; 293: 1323-1326. https://doi.org/10.1126/science.1062074 
[29] SKODA AM, SIMOVIC D, KARIN V, KARDUM V, VRANIC $S$ et al. The role of the Hedgehog signaling pathway in cancer: A comprehensive review. Bosn J Basic Med Sci 2018; 18: 8-20. https://doi.org/10.17305/bjbms.2018.2756

[30] ZHANG F, REN CC, LIU L, CHEN YN, YANG L et al. SHH gene silencing suppresses epithelial-mesenchymal transition, proliferation, invasion, and migration of cervical cancer cells by repressing the hedgehog signaling pathway. J Cell Biochem 2018; 119: 3829-3842. https://doi.org/10.1002/ jcb.26414

[31] ISLAM SS, MOKHTARI RB, NOMAN AS, UDDIN M, RAHMAN MZ et al. Sonic hedgehog (Shh) signaling promotes tumorigenicity and stemness via activation of epithelial-to-mesenchymal transition (EMT) in bladder cancer. Mol Carcinog 2016; 55: 537-551. https://doi.org/10.1002/ mc. 22300
[32] YOO YA, KANG MH, LEE HJ, KIM BH, PARK JK et al. Sonic hedgehog pathway promotes metastasis and lymphangiogenesis via activation of Akt, EMT, and MMP-9 pathway in gastric cancer. Cancer Res 2011; 71: 7061-7070. https:// doi.org/10.1158/0008-5472.CAN-11-1338

[33] XU X, SU B, XIE C, WEI S, ZHOU Y et al. Sonic hedgehogGlil signaling pathway regulates the epithelial mesenchymal transition (EMT) by mediating a new target gene, S100A4, in pancreatic cancer cells. PLoS One 2014; 9: e96441. https:// doi.org/10.1371/journal.pone.0096441

[34] ZHUANG Z, WANG K, CHENG X, QU X, JIANG B et al. LKB1 inhibits breast cancer partially through repressing the Hedgehog signaling pathway. PLoS One 2013; 8: e67431. https://doi.org/10.1371/journal.pone.0067431 


\section{Liver kinase B1 suppresses metastasis and angiogenesis of lung cancer: involvement of the Shh signaling pathway}

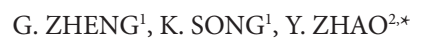

Supplemental material
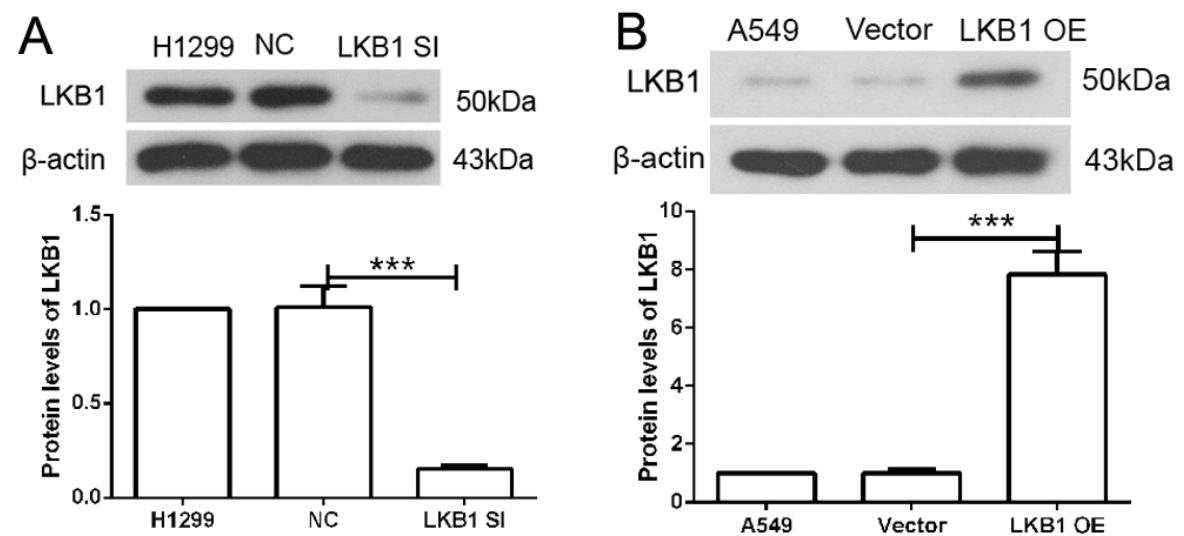

C
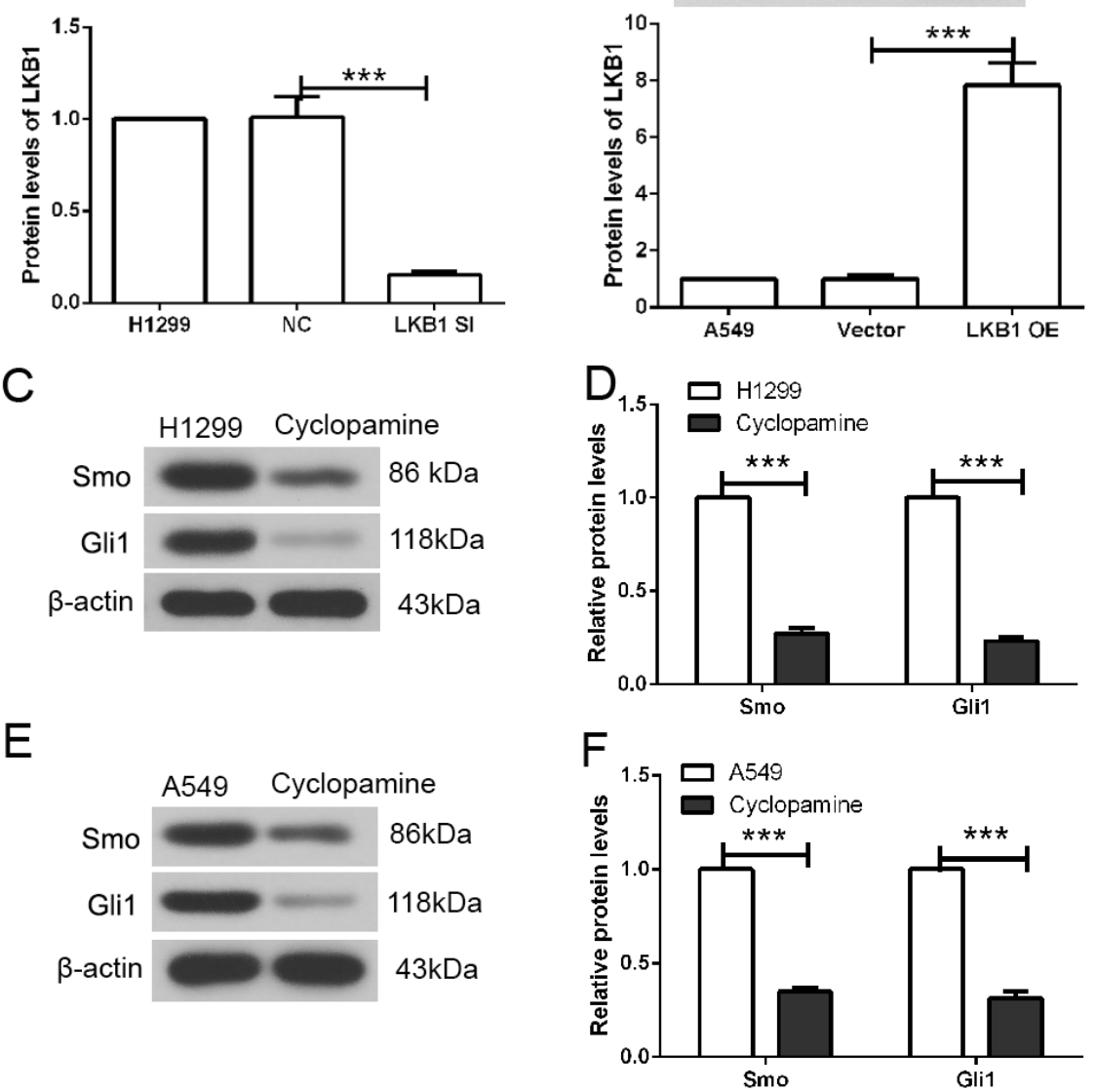

Supplementary Figure 1. Efficiency of LKB1 silence and LKB1 overexpression. A) After transfection with LKB1 shRNA, the levels of LKB1 in H1299 cells were measured by Western blot with $\beta$-actin as the internal control. B) Level of LKB1 in A549 cells was measured by Western blot assay after transfection with LKB1 overexpression plasmid. $\beta$-actin served as the internal control. C-F) The protein levels of Smo and Gli1 in H1299 cells and A549 cells were detected by western blot assay after treatment with cyclopamine. All experiments were repeated for three times. The results were presented as mean \pm SD. ${ }^{* * *} p<0.001$. LKB1 SI, LKB1 silence; NC, negative control of LKB1 silence; LKB1 OE, LKB1 overexpression; Vector, negative control of LKB1 overexpression. 